\title{
Research on Persuasive Design of Waste Sorting APP
}

\author{
Yuan $\mathrm{Hao}^{1,}$,, $\mathrm{Xu}$ Xiaoyan*1, b \\ ${ }^{1}$ Jiangsu University, Zhenjiang, 212000, China
}

\begin{abstract}
China's domestic waste classification policy is gradually being implemented, so that the domestic waste classification and processing industry is also developing rapidly and showing new characteristics of the digital age. Mobile phone application to assist urban residents in decision-making is one of the digital solutions for domestic waste classification. This article uses research methods such as literature research, case analysis and user interviews to sort out the characteristics and problems of existing waste sorting APPs, and analyze the characteristics of user waste sorting behavior. Then summarized based on the theory of persuasion, and finally proposed the persuasion design strategy of waste sorting APP from the three dimensions of motivation, ability, and triggering factors. It is expected that while satisfying the needs of users for assisting in waste sorting operations and optimizing user experience, it can further guide users to enhance their awareness of environmental protection and public welfare, participate in environmental protection public welfare activities, and bring better social benefits.
\end{abstract}

\section{Introduction}

With the steady growth of the economy, the acceleration of the pace of urbanization and the improvement of commodity circulation, the total amount of waste produced by urban residents of our country each year is increasing. In 2019 alone, the total output of domestic waste has reached 179 million tons. Despite the continuous improvement of the decontamination capacity of domestic waste, there is still a large amount of untreated domestic waste in cities, and the total amount of untreated waste in 2019 has reached 17 million tons [1]. Waste that is not collected and recycled will have a significant negative impact on the health of the population, public health and the ecological environment. In order to improve the efficiency of waste collection and disposal, on the one hand, the government should play the leading role in establishing a scientific and effective waste collection and disposal system, and on the other hand, residents should actively participate in cooperation to cultivate good habits of waste sorting and disposal, which is beneficial to the operation of the waste sorting and recycling system, can also reduce the amount of domestic waste from the source and reduce the output of domestic waste.

According to the requirements of the Implementing Plan for the Living Waste sorting System issued by the National Development and Reform Commission and the Ministry of Housing and Urban-rural Development in 2017, "by the end of 2020, basically establish the laws, regulations and standard systems relating to waste sorting, and form a replicable and propagable living waste sorting mode" [2]. On July 1, 2019, the Regulations of Shanghai Municipality on the Administration of Domestic Waste officially took effect[3],kicking off the nationwide

a285817681@qq.com

b*806935903@qq.com mandatory implementation of waste sorting policy. Since then, waste sorting related products have mushroomed in the market, but also included a lot of waste sorting APP. From the perspective of persuasive design theory, this paper will explore the interaction design strategy of waste sorting APP. In interaction design, the use of persuasive design is an important way to achieve this goal of behavioral change. Persuasive design, as a design approach that uses persuasion and social influence (not forcing) to change users' attitudes or behaviors, has the characteristics of sustainable development and plays a significant role in areas such as sports health, public health [4].

\section{Persuasive design and waste sorting APP}

\subsection{Persuasive design and its theoretical overview}

Sustainable interactive design is essentially the design of user behavior[5], and one of the most extensive ways to drive behavior change is to introduce persuasion technology into design, that is, to adopt persuasive design. The concept of persuasion technology was first proposed by Professor Fogg of Stanford University, developed developed from the field of computer technology [6], and then applied in many fields and then applied in a number of fields such as communication sales, political religion, cultural education, health and hygiene, the current domestic persuasion design in academic research is more commonly seen in sports health, online classroom and other fields.

Persuasive design, that is, the use of non-coercive way 
to change the theoretical method of user behavior, after many years of development and improvement, formed a series of theoretical achievements, including Fogg Behavior Model, Fogg Behavior Grid and PSD theory. Among them, Professor Fogg's behavior model provides an easier and more effective way for designers to analyze and design user behavior. As shown in Figure 1, the FBM behavior model suggests that user behavior consists of three elements -- motivation, ability, and trigger -- that behavior can occur only if it meets all three at the same time. This paper will also refer to the FBM to explore the persuasive design strategy in the waste sorting APP.

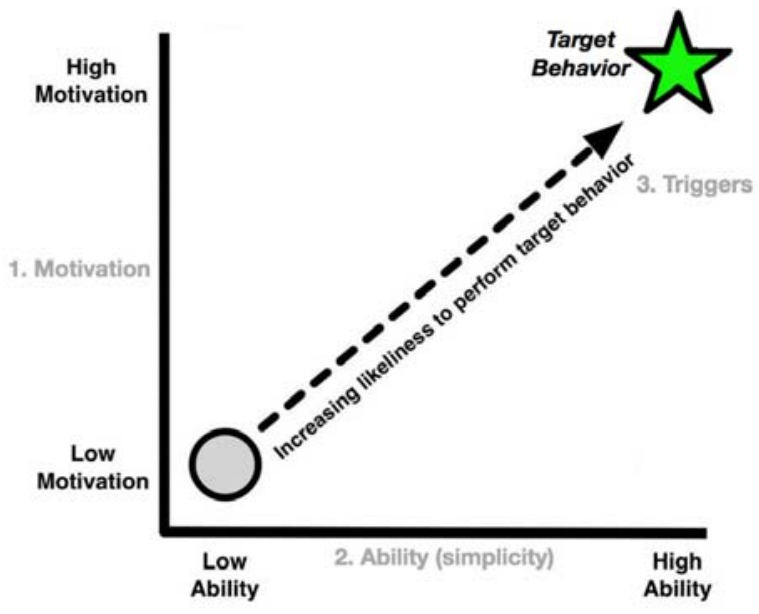

Figure 1. Fogg Behavior Model

Table 1. Overview of existing waste sorting apps on the Android platform

\begin{tabular}{lll}
\hline Sample number & Name. & Key features \\
\hline 1 & Waste Sorting & $\begin{array}{l}\text { Text entry search, search history, basic classification } \\
\text { instructions, delivery requirements, etc }\end{array}$ \\
3 & Waste Sorting & $\begin{array}{l}\text { Text input search, popular search tabs, basic category } \\
\text { descriptions, and delivery requirements, etc }\end{array}$ \\
5 & Waste Sorting & $\begin{array}{l}\text { Text input search, basic classification instructions, and } \\
\text { delivery requirements, Answer test, specific classification } \\
\text { of six common complex items, etc }\end{array}$ \\
delivery requirements, common items classification, etc
\end{tabular}

\section{Waste sorting and waste sorting APPs from the user's perspective}

Taking into account that young people are often the first to come into contact with new mobile phone applications, and this part of the population is also the main force responsible for the classification of domestic waste, the respondents of this questionnaire are mainly young people
(Picture source: www. BehaviorModel.org)

\subsection{Analysis of existing waste sorting APPs' features}

Currently waste sorting-related APPs, on Android and iOS platforms, are divided into two main categories, one for the guide tool, the other for puzzle games. Puzzle games on the theme of waste sorting are used less frequently in the daily lives of residents, and the target users are mostly children, so there is little discussion in this paper. The " waste sorting APP" discussed in this paper is a mobile phone application that assists residents to search for the results of waste sorting in their daily life.

Table 1 shows an overview of the five waste sorting APPS on the Android platform. It is found that the unified characteristics of these APPs' functions are: (1) simple function, (2) rough visual design, (3) serious homogenization. 
lowest level of understanding, 5 means the highest level of understanding) to ask the survey respondents about the knowledge of waste sorting. In terms of understanding the benefits of domestic waste classification, the survey respondents are equally divided into 3.91. Most survey respondents believe that they are relatively or very familiar with the benefits of domestic waste classification (36.17\% and $31.91 \%$ respectively), but they have an intermediate understanding of the rules of domestic waste classification. The score is 3.34 , that is, most people $(44.68 \%)$ believe that they have an intermediate level of understanding of the classification rules for domestic waste.

The survey results show that for the survey respondents who have not yet enforced the waste sorting policy in their location, they have not yet formed the habit of actively classifying domestic waste. Most people think that 'do not classify, just throw a bag directly to the waste collection point' is more consistent $(28.57 \%)$ Or generally conform to $(35.71 \%)$ their own habits, but for hazardous waste, basically the survey respondents $(85.72 \%)$ have the awareness of separate disposal. In contrast, the awareness of separate disposal of recyclable waste is low $(67.86 \%)$. For the survey respondents who have enforced the waste classification policy in their locations, $57.89 \%$ of them will sort and place the waste immediately after it is generated, and $21.05 \%$ will choose to accumulate a certain amount of waste and use their free time to sort again. After the waste is brought to the concentration point, there is less sorting on the spot, accounting for about $10 \%$. When the survey respondents encountered problems in the process of waste classification, they almost all chose to use mobile phones, computers, etc. for network retrieval (84.21\%), and most of them chose search engines such as Baidu. The survey respondents pointed out that in the process of using The main problems encountered include 'not detailed enough', 'easy to lose focus', 'some waste cannot be retrieved', and 'too complicated'. In the final survey on the willingness and expectation of waste sorting products, free APP tools were more favored by the survey respondents (average score 4.11), followed by ordinary sorting trash cans and other daily necessities (3.66 points), and then smart Sorting trash cans, kitchen waste disposers and other small appliances (3.23 points).

According to the results of the questionnaire survey, it is easy to find: (1) The survey subjects generally have a certain degree of understanding of the meaning and rules of household waste sorting. Mandatory policies and regulations are currently the main driving force to improve the implementation of waste classification behavior, and positive incentives The methods are relatively rare; (2) Combined with the time node when the household waste sorting behavior occurs, free APP products are more popular than smart products because of their convenience, efficiency and low cost; (3) The current waste sorting APPs on the market are not perfect, there is no relatively mature and highly accepted product.

\section{The persuasive design strategy of the waste sorting APP}

Combined with the user interview situation of urban residents, as well as the analysis of the current market waste sorting APP, the author put foward strategy and recommendations for the waste sorting APP persuasion design based on the theory of persuasive design, from the motivation, ability and trigger factors three angles.

\subsection{Persuasion strategies to strengthen user motivation}

There are three core drivers of motivation in the FBM behavior model, all of which are two-sided, namely pleasure and pain, hope and fear, social acceptance and social rejection[7]. Pleasure and pain are people's immediate reactions to things, people tend to produce pleasure and avoid pain, hope and fear are people's expectations of the future outcome of things, good things lead to hope and bad things lead to fear, social acceptance and rejection are the norm of social behavior of human beings and all groups of animals, people always tend to make themselves socially accepted rather than socially rejected. There is no difference between the three core dynamics, strength and weakness. Therefore, strengthening the user's motivation for waste sorting requires a pleasant experience, good outcome expectations, and social acceptance.

1) Optimize the overall visual design and enhance the user's sensory experience

Visual design includes all the visual factors of APP interface, such as color, graphics, layout, dynamics and text, which affect the aesthetic experience of the user both emotionally and rationally[8]. On the one hand, the beautiful interactive interface is conducive to attract users to understand and then contact the waste sorting APP, and in the subsequent use of the establishment of a holistic, good psychological image. On the other hand, reasonable visual introduction is conducive to the user to better understand the information, reduce the cognitive burden of the user. Therefore, the overall visual design of the spam classification APP needs to be optimized and enhanced with the goal of bringing pleasant aesthetic feeling and clear information characteristics.

2) Clear classification of processing results, strengthen the user's social motivation

The behavior of user participation in waste sorting itself has strong social attributes. At present, the main social motivation of most residents comes from the government's policy regulations and vague awareness of environmental protection. In fact, the government, schools, environmental protection associations and other organizations and individual environmental protection volunteers have done a lot of publicity and popularization work, but when residents actually carry out waste sorting work, compared to the long-term social, economic, ecological effects, cumbersome classification work is the most real experience at the time. Waste sorting APP can take on the auxiliary classification work at the same time, for the user to show the waste sorting and recycling after 
the image results. For example, after a user has completed the classification of plastic products using APP, the APP can prompt the user that "the plastic bottle you classify will have the opportunity to regenerate into a piece of clothing" or "The plastic bag you classify reduces the risk of a turtle eating by mistake". This approach not only brings good result expectations for the user's waste sorting behavior, but also clarifies the specific behavior that the user can obtain social acceptance.

\subsection{Persuasion strategies to enhance the user's capabilities}

1) Reduce the difficulty of the initial task and simplify the user process

The most initial, basic and core function of the waste sorting APP is to help users to sort waste, at the same time, the act of increasing waste sorting itself has been to the residents of our country accustomed to "throwing a bag" to increase the process of discarding waste. Therefore, the design of waste sorting APP first needs to reduce the initial task difficulty of waste sorting APP, matching the operational capabilities of most users, open app even if it can be operated. Secondly, it is necessary to simplify the operation of other derivative functions from the process, and reduce the ability of subsequent users to actively participate in further environmental public welfare activities.

Reduce the difficulty of the initial task, specifically, can be in the waste sorting APP home page to present the search method, combined with big data, to provide users with specific items, popular search items and even historical search items of direct labels. On the other hand, it is necessary to increase the choice of retrieval methods, such as voice input, image recognition and even AR scanning, to reduce the technical difficulty of the initial task. Simplifying the user process requires a reasonable information architecture that increases interface functionality while reducing operational steps, thereby reducing the user's operational time and effort consumption. As shown in figure 3 in Table 1, the results of the classification of thematic items are provided to the user.

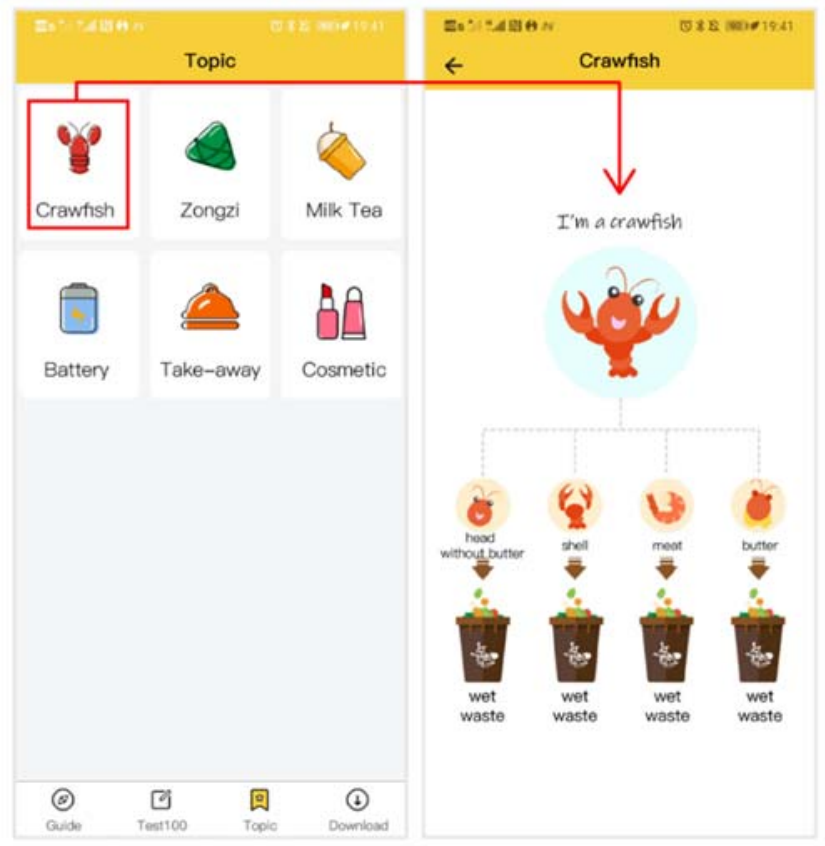

Figure 2. Sample 3 interface

(Picture source: Waste Sorting APP Screenshot)

2). Differentiated functional services to reduce user learning costs

In the national environment of waste sorting, each city and region has different waste sorting regulations adapted to local conditions. Therefore, the waste sorting APP needs a strong and complete database as a support, and in the user's knowledge, access to location information, for the user to match the local waste sorting information. In China's current high population mobility of the national conditions, beneficial to users in the production of location changes, reduce the cost of learning the new classification of time and energy, faster adaptation and integration into local life.

As for the individual user, the waste sorting APP can be based on the user's preferences, can more accurately match the user's classification situation, but also provide users with a fuller choice of operation space, directly filter the user's unwanted content and functions. For example, when a user has been classified for waste for a branded product, the results of the waste sorting associated with other products of the same brand or similar products of different brands are to be viewed by the user.

\subsection{Persuasion strategy to increase triggers}

\section{1). Give instant task feedback and provide easy task reminders}

Feedback itself is one of the most direct and important ways to establish a connection between the user and the APP, directly affecting the formation of the user's viscosity. The most direct embodiment is a variety of simple but popular mobile phone games, such as WeChat mini-game 'jump', is essentially the simplest click operation, but the game on the user's every click of the operation has made instant feedback, and according to the time of finger stay to change the feedback results.

The most basic function of waste sorting APP is to 
assist users to complete the behavior of waste sorting, as this behavior is repeated, users will continue to accumulate knowledge and experience of waste sorting, thereby gradually reducing the use of APP. Therefore, reasonable user feedback can not only help users to achieve a sense of accomplishment in the completion of basic tasks, but also trigger the user's use of Yansheng function, encourage users to learn more about environmental protection, participate in environmental protection activities, cultivate environmental protection habits. Proper feedback can improve the user's motivation and avoid psychological oppression on the user. As shown in the figure 3, sample 1 in Table 1 , in the home page shows the children's paintings, not only to publicity of waste sorting, but also to participate in the children's encouragement. What's more, it is going to encourage more children to participate.

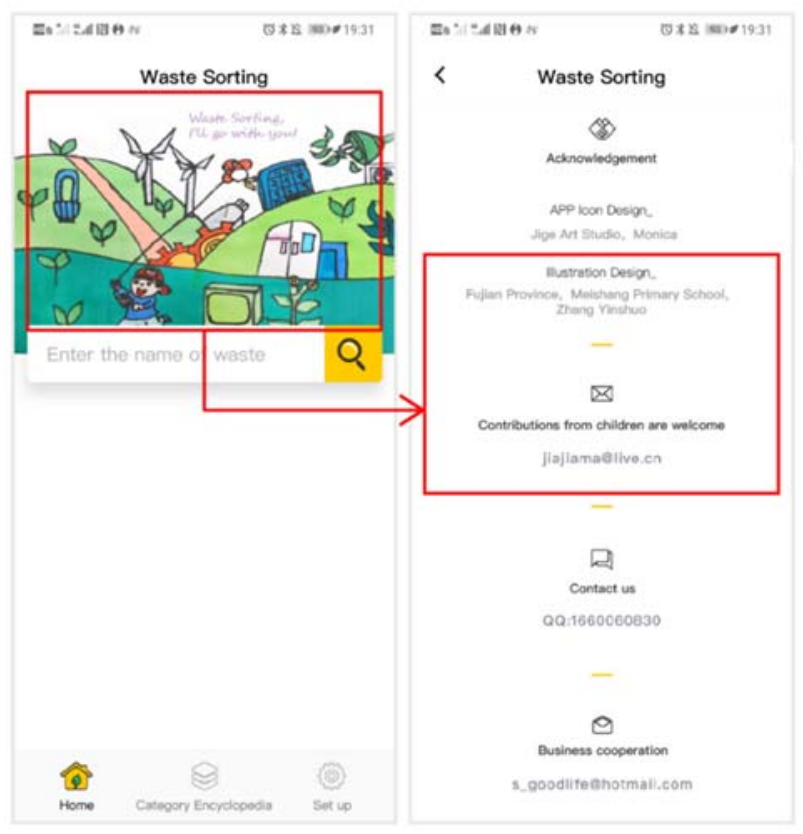

Figure 3. Sample 1 interface

(Picture source: Waste Sorting APP Screenshot)

2). Establish an honor achievement system and set up moderate social participation

The purpose of establishing the honor achievement system is to stimulate users to participate in waste sorting and related environmental public welfare activities by setting corresponding rewards, which can include virtual currency, status level, certificate of honor and medal titles. Setting up moderate social engagement in a spam-sorting APP can enhance the incentive for users to achieve honorable achievements. The use of forms of social participation, such as competition, comparison, and cooperation, is a more common persuasive design strategy [9].

\section{Conclusion}

Although the current release of waste sorting APP is still relatively simple in general, but with the gradual promotion of the national waste sorting related policy provisions, and the trend of industrialization of the waste treatment industry, waste sorting APP as a member of the waste sorting treatment products, there is a very large space for development. Persuasive design can provide more systematic theoretical support and method guidance for the interactive design of waste sorting APP. Based on the FBM behavior model, the author provides the interactive design strategy and suggestion of spam classification APP from the three dimensions of motivation, ability and trigger factors, not only hope to optimize the interactive design of spam classification APP, so as to enhance the user experience, but also hope to promote users to learn more environmental public welfare knowledge and participate in more environmental public welfare activities.

\section{References}

1. China Report Hall. (2020) Household waste disposal industry statistics.www.chinabgao.com /freereport/81722.html.

2. National Development and Reform Commission, housing urban and rural areas. (2017) The Ministry of Construction's Life Classification System Implementation Program.

3. Member of the Standing Committee of the Shanghai Municipal People's Congress. (2019) Shanghai Municipal Regulations on the Administration of Household Waste.

4. Bremer C. (2020) Not (B)interested? Using Persuasive Technology to Promote Sustainable Household Recycling Behavior. In: The 15th International Conference on Persuasive Technology, Aalborg.pp.195-207.

5. Xin XY. (2015) Interactive Design: From Physical Logic to Behavioral Logic. Decoration, 01:58-62.

6. Fogg B J. (2002) Persuasive Technology: Using Computers to Change What We Think and Do. Ubiquity, 9: 1-30.

7. Fogg B J. (2009) A Behavior Model for Persuasive Design. In:The 4th International Conference on Persuasive Technology, California. pp. 1-7.

8. Yuan H, et al. (2020) Motion APP Information Visual Interface Design Research. Packaging Engineering ,41(18): 236-241.

9. Orji R. (2017) Why Are Persuasive Strategies Effective? Exploring the Strengths and Weaknesses of Socially-Oriented. In: The 12th International Conference on Persuasive Technology, Amsterdam. pp. 253-266. 